\title{
Rezensionen online
}

\section{... von der Redaktion betreut (Dezember 2018 - Februar 2019)}

Die Redaktion der Vierteljahrshefte für Zeitgeschichte arbeitet seit 2003 im Auftrag des Instituts für Zeitgeschichte München-Berlin mit dem Rezensionsjournal sehepunkte zusammen. Diese Kooperation findet nicht nur in den sehepunkten ihren Niederschlag, sondern auch in den Vierteljahrsheften selbst: In jedem Heft werden die von der Redaktion angeregten und betreuten Rezensionen angezeigt, die in den drei Monaten zuvor in den sehepunkten erschienen sind.

Alex Aßmann, Gudrun Ensslin. Die Geschichte einer Radikalisierung, Paderborn 2018.

Rezensiert von: Martin Jander (Berlin) in sehepunkte 18 (2018), Nr. 12

www.sehepunkte.de/2018/12/32191.html

Stefan Creuzberger / Fred Mrotzek / Mario Niemann (Hgg.), Land im Umbruch. Mecklenburg-Vorpommern nach dem Ende der DDR, Berlin 2018.

Rezensiert von: Eva Lütkemeyer (Institut für Zeitgeschichte München-Berlin) in sehepunkte 19(2019), Nr. 2

www.sehepunkte.de/2019/02/31384.html

Jost Dülffer, Geheimdienst in der Krise. Der BND in den 1960er-Jahren, Berlin 2018.

Rezensiert von: Heiner Möllers (Zentrum für Militärgeschichte und Sozialwissenschaften der Bundeswehr, Potsdam) in sehepunkte 19 (2019), Nr. 1

www.sehepunkte.de/2019/01/31648.html

Mark Haarfeldt, Deutsche Propaganda im Rheinland 1918-1936, Essen 2017.

Rezensiert von: Wolfgang Elz (Mainz) in sehepunkte 19 (2019), Nr. 2

www.sehepunkte.de/2019/02/32019.html

Ludwik Hirszfeld, Geschichte eines Lebens. Autobiographie, Paderborn 2018.

Rezensiert von: Benedikt Faber (Gymnasium Augustinianum, Greven) in sehepunkte 18 (2018), Nr. 12

www.sehepunkte.de/2018/12/32278.html

Jason B. Johnson, Divided Village. The Cold War in the German Borderlands, London / New York 2017.

Rezensiert von: Roger Engelmann (Abteilung Bildung und Forschung bei dem Bundesbeauftragten für die Unterlagen des Staatssicherheitsdienstes der ehemaligen DDR, Berlin) in sehepunkte 19 (2019), Nr. 1

www.sehepunkte.de/2019/01/31600.html 
Pieter M. Judson, Habsburg. Geschichte eines Imperiums 1740-1918, München 2017.

Rezensiert von: Wolfgang Elz (Mainz) in sehepunkte 18 (2018), Nr. 12

www.sehepunkte.de/2018/12/31832.html

Jürgen Kilian, Krieg auf Kosten anderer. Das Reichsministerium der Finanzen und die wirtschaftliche Mobilisierung Europas für Hitlers Krieg, Berlin / Boston 2017. Rezensiert von: Paul-Moritz Rabe (NS-Dokumentationszentrum München) in sehepunkte 19 (2019), Nr. 2

www.sehepunkte.de/2019/02/31718.html

Jakob Knab, Ich schweige nicht. Hans Scholl und die weiße Rose, Darmstadt 2018. Rezensiert von: Christiane Moll (München) in sehepunkte 19 (2019), Nr. 1 www.sehepunkte.de/2019/01/31413.html

Carl Alexander Krethlow, Bagdad 1915/17. Weltkrieg in der Wüste, Paderborn 2018.

Rezensiert von: Bernd Lemke (Zentrum für Militärgeschichte und Sozialwissenschaften der Bundeswehr, Potsdam) in sehepunkte 19 (2019), Nr. 2

www.sehepunkte.de/2019/02/32092.html

Neill Lochery, Out of the Shadows. Portugal from Revolution to the Present Day, London 2017.

Rezensiert von: Christiane Abele (Paris) in sehepunkte 18 (2018), Nr. 12

www.sehepunkte.de/2018/12/31562.html

Leonid Luks, Totalitäre Versuchungen. Russische Exildenker über die Ursachen der russischen Revolution und über den Charakter der europäischen Krise des 20. Jahrhunderts, Münster u. a. 2017.

Rezensiert von: Jutta Scherrer (École des hautes études en sciences sociales, Paris) in sehepunkte 19 (2019), Nr. 1

www.sehepunkte.de/2019/01/31779.html

Titus J. Meier, Widerstandsvorbereitungen für den Besetzungsfall. Die Schweiz im Kalten Krieg, Zürich 2018.

Rezensiert von: Thomas Riegler (Wien) in sehepunkte 19 (2019), Nr. 1

www.sehepunkte.de/2019/01/32181.html

Giovanni Messe, Lettere alla moglie. Dai fronti greco-albanese, russo, tunisino e dalla prigionia 1940-1944, Mailand 2018.

Rezensiert von: Thomas Schlemmer (Institut für Zeitgeschichte München-Berlin) in sehepunkte 19 (2019), Nr. 2

www.sehepunkte.de/2019/02/32806.html 
Jann Müller, Die Wiederbegründung der Industrie- und Handelskammern in Ostdeutschland im Prozess der Wiedervereinigung, Stuttgart 2017.

Rezensiert von: Max Trecker (Institut für Zeitgeschichte München-Berlin) in sehepunkte 19 (2019), Nr. 2

www.sehepunkte.de/2019/02/30998.html

Jürgen Nielsen-Sikora, Hans Jonas. Für Freiheit und Verantwortung, Darmstadt 2017.

Rezensiert von: Michael Gehler (Hildesheim) in sehepunkte 18 (2018), Nr. 12

www.sehepunkte.de/2018/12/30717.html

Kiran Klaus Patel, Projekt Europa. Eine kritische Geschichte, München 2018.

Rezensiert von: Guido Thiemeyer (Heinrich-Heine-Universität, Düsseldorf) in sehepunkte 19 (2019), Nr. 2

www.sehepunkte.de/2019/02/32441.html

Julia Paulus (Hg.), „Bewegte Dörfer“. Neue soziale Bewegungen in der Provinz 1970-1990, Paderborn 2018.

Rezensiert von: Anne Bieschke (Universität Mannheim) in sehepunkte 19 (2019), Nr. 2

www.sehepunkte.de/2019/02/32070.html

Kim Phillips-Fein, Fear City. New York's Fiscal Crisis and the Rise of Austerity Politics, New York 2017.

Rezensiert von: Christian Rau (Institut für Zeitgeschichte München-Berlin) in sehepunkte 19 (2019), Nr. 2

www.sehepunkte.de/2019/02/32201.html

Moritz Pöllath, Eine Rolle für die NATO out-of-area? Das Bündnis in der Phase der Dekolonisierung 1949-1961, Frankfurt/M. u. a. 2017.

Rezensiert von: Gerhard Altmann (Korb) in sehepunkte 18 (2018), Nr. 12

www.sehepunkte.de/2018/12/31632.html

Felix Römer, Die narzisstische Volksgemeinschaft. Theodor Habichts Kampf 1914 bis 1944, Frankfurt/M. 2017.

Rezensiert von: Christian Packheiser (Institut für Zeitgeschichte München-Berlin) in sehepunkte 18 (2018), Nr. 12

www.sehepunkte.de/2018/12/29703.html

Stefan Scholl (Hg.), Körperführung. Historische Perspektiven auf das Verhältnis von Biopolitik und Sport, Frankfurt/M. / New York 2018.

Rezensiert von: Frank Becker (Universität Duisburg-Essen) in sehepunkte 19 (2019), Nr. 1 www.sehepunkte.de/2019/01/32068.html 
Alexander Sedlmaier, Konsum und Gewalt. Radikaler Protest in der Bundesrepublik, Berlin 2018.

Rezensiert von: Eckhard Jesse (Technische Universität, Chemnitz) in sehepunkte 19 (2019), Nr. 2 www.sehepunkte.de/2019/02/32813.html

Konstanze Soch, Eine große Freude? Der innerdeutsche Paketverkehr im Kalten Krieg (1949-1989), Frankfurt/M. / New York 2018.

Rezensiert von: Christoph Lorke (Westfälische Wilhelms-Universität, Münster) in sehepunkte 18 (2018), Nr. 12

www.sehepunkte.de/2018/12/32264.html

Sybille Steinbacher (Hg.), Rechte Gewalt in Deutschland. Zum Umgang mit dem Rechtsextremismus in Gesellschaft, Politik und Justiz, Göttingen 2016.

Rezensiert von: Richard Büttner (München) in sehepunkte 19 (2019), Nr. 1

www.sehepunkte.de/2019/01/29570.html

Alina Laura Tiews, Fluchtpunkt Film. Integrationen von Flüchtlingen und Vertriebenen durch den deutschen Nachkriegsfilm 1945-1990, Berlin 2017.

Rezensiert von: Andreas Kötzing (Hannah-Arendt-Institut für Totalitarismusforschung e.V. an der Technischen Universität, Dresden) in sehepunkte 18 (2018), Nr. 12

www.sehepunkte.de/2018/12/30891.html

Mario Wenzel, Arbeitszwang und Judenmord. Die Arbeitslager für Juden im Distrikt Krakau des Generalgouvernements 1939-1944, Berlin 2017.

Rezensiert von: Frank Grelka (Europa-Universität Viadrina, Frankfurt/Oder) in sehepunkte 19 (2019), Nr. 2

www.sehepunkte.de/2019/02/32296.html

Rüdiger Wenzke, Wo stehen unsere Truppen? NVA und Bundeswehr in der ČSSRKrise 1968, Berlin 2018.

Rezensiert von: Christian Th. Müller (Universität Potsdam) in sehepunkte 19 (2019), Nr. 1 www.sehepunkte.de/2019/01/32281.html

Jan Ole Wiechmann, Sicherheit neu denken. Die christliche Friedensbewegung in der Nachrüstungsdebatte 1977-1984, Baden-Baden 2017.

Rezensiert von: Dimitrij Owetschkin (Bochum) in sehepunkte 19 (2019), Nr. 1

www.sehepunkte.de/2019/01/29323.html

Oliver von Wrochem (Hg.), Nationalsozialistische Täterschaften. Nachwirkungen in Gesellschaft und Familie, Berlin 2016.

Rezensiert von: Niels Weise (Institut für Zeitgeschichte München-Berlin) in sehepunkte 18 (2018), Nr. 12

www.sehepunkte.de/2018/12/29247.html 
Tim-Lorenz Wurr, Terrorismus und Autokratie. Staatliche Reaktionen auf den Russischen Terrorismus 1870-1890, Paderborn 2017.

Rezensiert von: Vitalij Fastovskij (München) in sehepunkte 18 (2018), Nr. 12

www.sehepunkte.de/2018/12/31297.html

Robert M. Zoske, Flamme sein! Hans Scholl und die Weiße Rose. Eine Biografie, München 2018.

Rezensiert von: Christiane Moll (München) in sehepunkte 19(2019), Nr. 1

www.sehepunkte.de/2019/01/31413.html

Rezension zu Publikationen des IfZ (Dezember 2018 - Februar 2019):

Martina Steber, Die Hüter der Begriffe. Politische Sprachen des Konservativen in Großbritannien und der Bundesrepublik Deutschland, 1945-1980, Berlin / Boston 2017.

Rezensiert von: Nikolai Wehrs (Universität Konstanz) in sehepunkte 18 (2018), Nr. 12 www.sehepunkte.de/2018/12/30931.html 


\section{DE DE GRUYTER OLDENBOURG}

\section{GERMAN YEARBOOK OF CONTEMPORARY HISTORY}

\section{VOLUME 3}

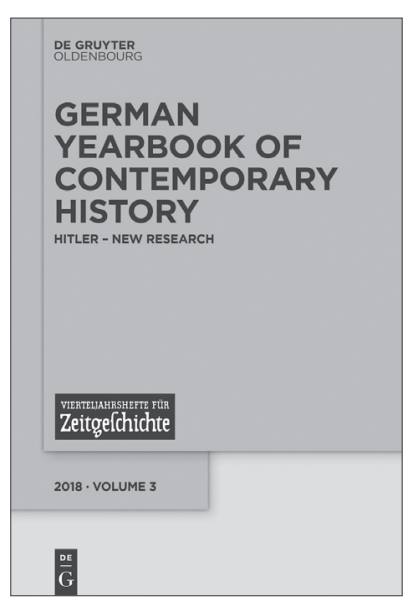

Elizabeth Harvey, Johannes Hürter (Eds.)

\section{HITLER - NEW RESEARCH}

2018. 244 pages

HC RRP $€ 49.95[D] / U S \$ 57.99 / £ 45.50$

ISBN 978-3-11-055322-2

eBook RRP $€ 49.95[D] /$ US $\$ 57.99 / £ 45.50$

PDF ISBN 978-3-11-055560-8

ePUB ISBN 978-3-11-055346-8

How should we understand Hitler as a factor in the history of the Third Reich? Academic interest in the German dictator has been increasing once again, as shown by recent debates surrounding the publication of "Mein Kampf," and by numerous new studies on Hitler's personality, ideology and politics. Edited by Elizabeth Harvey (University of Nottingham) and Johannes Hürter (Institute of Contemporary History Munich - Berlin), the third volume of the German Yearbook of Contemporary History presents the latest in German research on Hitler based on selected articles from the Vierteljahrshefte für Zeitgeschichte. Novel theories concerning Hitler's personality and authenticity, the sources of his radical racism, and the relationship between the dictator and German society are assessed by renowned experts from the English-speaking world. 